\title{
18 Missbrauch anabol-androgener Steroide
}

\author{
Hans-Christian Schuppe
}

\begin{abstract}
Testosteron wird für die sexuelle Differenzierung sowie die Ausbildung des männlichen Phänotyps benötigt und beeinflusst Sexualverhalten und -aktivität beim Mann. Neben diesen androgenen Effekten hat Testosteron eine starke proteinaufbauende (anabole) Wirkung.

Bei Patienten mit einer Hodenunterfunktion und entsprechendem Androgenmangel bewirkt die Testosteronsubstitution eine Zunahme des Körpergewichts zugunsten der fettfreien Körpermasse (Bagatell u. Bremner 1996; s. Kap. Il.11). Die gesteigerte Muskelmasse ist auf ein Wachstum der Muskelfibrillen zurückzuführen und mit einer verbesserten Muskelkraft verbunden. Zusammen mit körperlichem Training erlauben supraphysiologische Testosteron-Gaben auch bei gesunden Männern eine Steigerung der Muskelmasse und -kraft (Bhasin et al. 1996).
\end{abstract}

\subsection{Anabol-androgene Steroide (AAS)}

In den vergangenen 6o Jahren wurden zahlreiche Modifikationen des Testosteronmoleküls (Mottram u. George 200o, s. Abb. 1) synthetisiert, um die rasche Metabolisierung von Testosteron in der Leber zu umgehen und geeignete Darreichungsformen für eine Substitutionsbehandlung zu erhalten. Andererseits zielten die Bemühungen darauf ab, Steroide mit überwiegend anaboler Wirkung und möglichst geringer androgener Komponente für geeignete medizinische Indikationen wie z.B. konsumierende Erkrankungen zu entwickeln, eine vollständige Trennung der beiden Wirkungen ist jedoch bisher nicht möglich.

\subsection{Anwendung und Missbrauch von AAS}

AAS wurden bereits sehr früh ohne medizinische Indikation im Spitzensport eingesetzt, um auf unphysiologische Weise die Leistungsfähigkeit zu steigern („Doping“; Verroken 200o; Sjöqvist et al. 2008). Erste Berichte über eine Anwendung von Testosteron-Derivaten durch Gewichtheber stammen aus den 1950er-Jahren. In den folgen- 


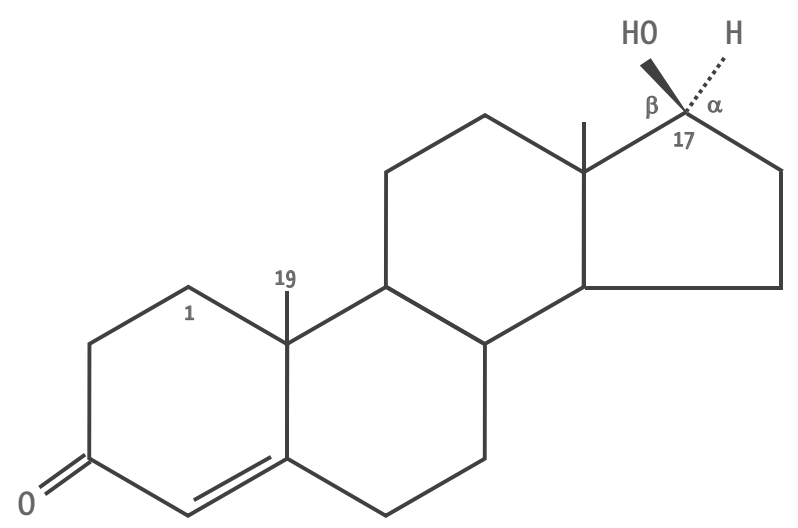

Abb. 1 Strukturformel von Testosteron. Gebräuchliche Modifikationen des Moleküls sind 17ß-Hydroxyl-Ester (z.B. Testosteron-Enanthat, Testosteron-Undecanoat), 17a-alkylierte Derivate (z.B. Methyltestosteron, Stanozolol), 1a-alkylierte Derivate (z.B. Methenolon, Mesterolon) sowie 19-Nortestosteron (Entfernung von C19; Nandrolon) und seine Derivate.

den Jahrzehnten hat der Missbrauch von AAS im Leistungssport erheblich zugenommen, wobei die Prävalenz im Bodybuilding und bei Gewichthebern am höchsten ist. Bemerkenswert ist in diesem Zusammenhang die ethisch nicht akzeptable Behandlung von Athleten in staatlich-geförderten Doping-Programmen wie z.B. in der ehemaligen DDR (Franke u. Berendonk 1997). Trotz frühzeitiger ärztlicher Warnungen, Berücksichtigung der AAS in Verboten des internationalen Olympischen Komitees (IOC) unmittelbar nach Etablierung geeigneter Nachweismethoden und Einführung von Doping-Kontrollen ist das Problem des AAS-Abusus im internationalen Wettkampfsport bis heute aktuell (Wade 1972; Basaria 2010). In den vom IOC akkreditierten Referenzlaboratorien sind ca. 1\% der jährlich untersuchten Proben positiv (Schänzer 2004). Für den Nachweis von AAS bzw. ihren Metaboliten werden Urinproben mittels Gaschromatografie und Massenspektrometrie analysiert.

\section{Der Missbrauch leistungssteigernder Medikamente hat längst den Breiten- und Freizeitsport erreicht.}

AAS zählen auch in der allgemeinen Bevölkerung zu den am häufigsten angewendeten leistungssteigernden Pharmaka (Dawson 2001; Sjöqvist et al. 2008). Einige Studien gehen von einer Prävalenz von bis zu 8o\% bei Männern im Bodybuilding aus, bis zu 25\% der Freizeitsportler in Fitness-Studios wenden AAS und andere leistungssteigernde Mittel an (Boos et al. 1998; Striegel et al. 2006). Unter Jugendlichen in den USA und Australien erhobene Daten zeigen für den AAS-Konsum eine Prävalenz von 3-12\% bei Jungen und 1-2\% bei Mädchen (Handelsman u. Gupta 1997; Bahrke et al. 200o). Britischen Daten zufolge finden sich AAS unter den Jugendlichen illegal angebotenen Substanzen an dritter Stelle nach Cannabis und Amphetaminen (Dawson 2001). Auch in bestimmten Berufsgruppen wie z.B. Angehörigen von Sicherheitsdiensten, Polizei- und Militäreinheiten ist vermehrt mit einem Missbrauch leistungssteigernder Pharmaka einschließlich AAS zu rechnen. 
Anabol-androgene Steroide (AAS) werden von Männern ohne medizinische Indikation eingenommen, um auf unphysiologische Weise die Leistungsfähigkeit im Sport zu steigern (,win at all costs") oder dem Wunsch nach Veränderung der Körperproportionen entsprechend Muskelmasse und -kraft aufzubauen (,win the battle of attractiveness").

In der täglichen Praxis wird von Patienten, die missbräuchlich AAS anwenden, seltener eine verbesserte Wettkampfleistung als vorrangiges Ziel angegeben, sondern der Aufbau von Muskelmasse und -kraft. Dieser Wunsch mancher Männer nach Veränderung ihrer Körperproportionen ist vor dem Hintergrund zu verstehen, dass sie sich in ihrer Selbstwahrnehmung eher als untergewichtig empfinden (Pope et al. 2000; Petersson et al. 2010). Die pathologische Beschäftigung mit einem eingebildeten Mangel an Muskelmasse wird als körperdysmorphe Störung („Biggerexia“, „Muskel-Dysmorphie“), d.h. somatoforme Störung nach DSM-IV (Diagnostisches und Statistisches Manual psychischer Störungen) aufgefasst. Von einer zunehmenden Zahl von Männern werden AAS als „Lifestyle“-Medikamente aufgefasst und in der Absicht angewendet, das Allgemeinbefinden zu verbessern (s. Kap. II.19) Nicht selten ist der AAS-Abusus mit dem Konsum von Rauschmitteln bzw. anderen Genussgiften wie Alkohol und Tabak assoziiert (Durant et al. 1993; Sjöqvist et al. 2008).

\section{Das gesteigerte Interesse am körperlichen Erscheinungsbild bei Bodybuildern ist mit demjenigen bei Essstörungen vergleichbar.}

\subsection{AAS-Missbrauch: Präparate und Dosierungen}

Im Rahmen des Missbrauchs kommt neben den in Deutschland zugelassenen und im Handel befindlichen Androgenen, insbesondere den intramuskulär zu applizierenden Depotpräparaten Testosteron-Enanthat und -Propionat sowie dem oral wirksamen Testosteron-Undecanoat, ein breites Spektrum anaboler Steroide zur Anwendung (Schänzer 2004, s. Tab. 1). Unter den am häufigsten eingesetzten Präparaten finden sich auch zahlreiche 17a-alkylierte AAS (s. Abb. 1), die aufgrund ihrer Nebenwirkungen für medizinische Indikationen seit langem obsolet sind. Darüber hinaus sind neuartige „Designer-Steroide“ wie Tetrahydrogestrinone zu berücksichtigen, die ohne jegliche pharmako-toxikologische Prüfung verbreitet und eingesetzt werden (Death et al. 2004). Zu den neueren Substanzen mit anaboler Wirkung gehören auch die sog. Selektiven Androgen-Rezeptor-Modulatoren (SARM). Bei der Untersuchung zur Leistungssteigerung angepriesener Nahrungsergänzungspräparate („nutriceuticals“) ließen sich in ca. $15 \%$ der Zubereitungen nicht deklarierte AAS in Doping-relevanten Mengen nachweisen (Geyer et al. 2004).

Der Bezug der genannten Präparate erfolgt vor allem über einen umfangreichen Schwarzmarkt, sowohl im Internet als auch in Fitness-Studios (nach einer anonymen Befragung im Internet in 70\% bzw. 25\% der Fälle; Parkinson u. Evans 2006). Verschiedene Studien belegen jedoch auch eine ärztliche Verordnung bzw. Abgabe durch Apotheken (inkl. Internet) in 15-20\% der Fälle (Boos et al. 1998; Parkinson u. Evans 2006; Striegel et al. 2006). Zu berücksichtigen sind darüber hinaus veterinärmedizinische Arzneimittel sowie insbesondere im Schwarzmarkthandel in ihrer Qualität und Reinheit nicht gesicherte, z.T. nicht näher identifizierbare Präparate. 


\section{Tab. 1 Anabole Steroide*}

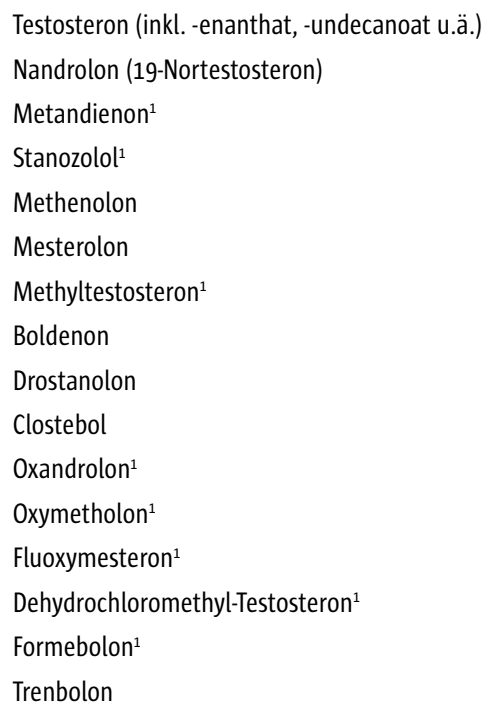

Die Einnahme- und Dosierungsschemata entsprechen nicht denjenigen bei klinischer Anwendung, sondern es erfolgt zumeist ein exzessiver Missbrauch, in der Vorstellung so ein möglichst rasches und starkes Muskelwachstum zu erreichen (Mottram u. George 2000; Sjöqvist et al. 2008). 60\% der Teilnehmer der o.g. Internet-basierten anonymen Befragung gaben an, mindestens 1.000 mg Testosteron bzw. äquivalente AAS pro Woche einzusetzen (Parkinson u. Evans 2006). Nach einschlägigen „Anleitungen“ (z.B. „Underground Steroid Handbook“; Duchaine 1989) werden Kombinationen verschiedener Präparate oral und intramuskulär in Schemata mit auf- und absteigenden Dosierungen („stacking“) über 4-18 Wochen („Kuren“) und sehr variablen behandlungsfreien Intervallen angewendet.

\section{Die Dosierungen bei AAS-Missbrauch überschreiten den für eine Testosteron- Substitutionstherapie bei Hypogonadismus erforderlichen Bereich um das 10-100-fache.}

Zur Verstärkung der Effekte bzw. zur Abmilderung AAS-bedingter Nebenwirkungen werden oft noch zusätzliche Medikamente wie z.B. Insulin, Wachstumshormon, Schilddrüsenhormone, humanes Choriongonadotropin (hCG), Anti-Östrogene oder Diuretika eingesetzt (Dawson 2001; Parkinson u. Evans 2006; Sjöqvist et al. 2008). Daten über die Auswirkungen derartiger Therapieregime auf das männliche Reproduktionssystem stehen naturgemäß kaum zur Verfügung. 
Tab. 2 Nebenwirkungen androgen-anaboler Steroide

\begin{tabular}{|c|c|}
\hline Hypothalamus-Hypophysen-Funktion* & $\begin{array}{l}\text { Suppression der Gonadotropinsekretion } \\
\text { vermindertes Hodenvolumen } \\
\text { Oligo- bis Azoospermie, Infertilität } \\
\text { Testosteronmangel } \\
\text { Libidoverlust }\end{array}$ \\
\hline Brustdrüsenkörper & Gynäkomastie \\
\hline Hämatopoese & $\begin{array}{l}\text { Polyglobulie } \\
\text { thrombembolische Komplikationen }\end{array}$ \\
\hline kardiovaskuläres System & Kardiomyopathie, plötzlicher Herztod \\
\hline Leber $^{1}$ & $\begin{array}{l}\text { intrahepatische Cholestase } \\
\text { Peliosis hepatis (hämorrhagische Leberzysten) } \\
\text { hepatozelluläre Adenome/Karzinome }\end{array}$ \\
\hline Stoffwechselfunktionen & $\begin{array}{l}\text { Verminderung HDL-, Anstieg LDL-Cholesterin } \\
\text { Hyperinsulinismus }\end{array}$ \\
\hline Skelettsystem & vorzeitiger Epiphysenfugenschluss (bei Adoleszenten) \\
\hline Haut & $\begin{array}{l}\text { Akne } \\
\text { Alopezie }\end{array}$ \\
\hline Psyche & $\begin{array}{l}\text { Stimmungsschwankungen } \\
\text { Depression, Psychosen (nach Absetzen) } \\
\text { gesteigerte Aggressivität (?) }\end{array}$ \\
\hline \multicolumn{2}{|c|}{$\begin{array}{l}\text { * Bei Frauen: Oligo- bis Amenorrhoe, Infertilität; Virilisierung mit männlicher Sekundärbehaarung, Absenkung de } \\
\text { Stimmlage, Klitorishypertrophie }\end{array}$} \\
\hline
\end{tabular}

AAS-Anwender schätzen das Wissen ihres Arztes über anabol-androgene Steroide geringer ein als dasjenige von Freunden und Dealern. Die Mehrzahl der Anwender verschweigt den Missbrauch gegenüber Ärzten.

\subsection{Nebenwirkungen der AAS}

Die wesentlichen Nebenwirkungen der AAS sind in Tabelle 2 aufgeführt. In der andrologischen Praxis ist vor allem die Suppression der Hypothalamus-Hypophysenfunktion mit entsprechend herabgesetzter endogener Testosteronproduktion, Libidoverlust und Erektionsstörungen sowie Unterdrückung der Spermatogenese bis hin zur Azoospermie und Hodenatrophie zu berücksichtigen.

Die Infertilität infolge einer hochgradigen Oligo- oder Azoospermie gilt nach Absetzen der AAS als reversibel, die Erholung der Spermatogenese kann jedoch über 6 Monate und länger dauern! 
Fälle einer irreversiblen Beeinträchtigung der Fertilität bzw. eines dauerhaften Hypogonadismus nach AAS-Abusus wurden beschrieben, bei Kinderwunsch sind hier eine Stimulationsbehandlung mit Gonadotropinen sowie ggf. Maßnahmen der assistierten Fertilisation erforderlich.

Neben der Infertilität sind im Rahmen des AAS-bedingten Hypogonadismus Libidoverlust und Erektionsstörungen zu berücksichtigen, insbesondere die aromatisierbaren, d.h. zu Östrogenen umwandelbaren Präparate induzieren häufig eine Gynäkomastie (s. Kap. II.12). An der Haut führt die Einnahme von AAS zu Seborrhoe, Talgdrüsenhyperplasie und vermehrter Besiedlung mit Propionibacterium acnes, die klinischen Krankheitsbilder reichen von der Akne papulopustulosa bis zur Akne fulminans (Melnik et al. 2007, s. Abb. 2).

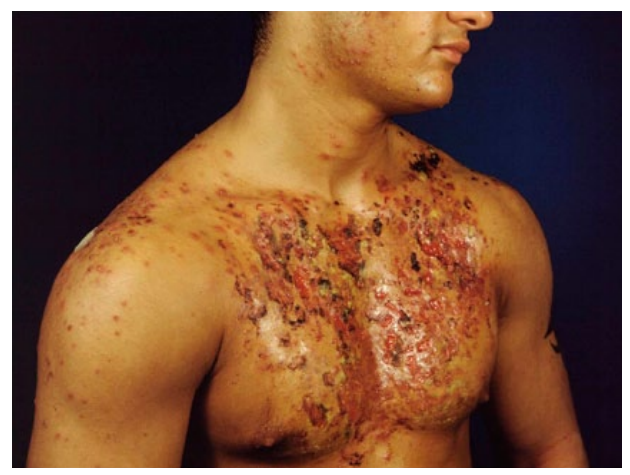

Abb. 2 Acne fulminans mit sternoklavikulären Knochenläsionen unter Missbrauch anabolandrogener Steroide (22-jähriger Patient) (Harth u. Gieler 2006, mit freundlicher Genehmigung von Springer Science and Business Media)

Eine erhebliche Hepatotoxizität einschließlich neoplastischer Prozesse ist mit der Anwendung $17 \alpha$-alkylierter Präparate verbunden (Bagatell u. Bremner 1996; Basaria 2010). Darüber hinaus sind Veränderungen im Lipidstoffwechsel sowie die Gefahr kardiovaskulärer und thrombembolischer Komplikationen zu beachten. Eine dosisabhängige Myokardhypertrophie fand sich bei Kraftsport-treibenden Männern mit AAS-Abusus, wobei der Effekt durch zusätzliche Gabe von Wachstumshormon verstärkt wurde (Karila et al. 2003). Neben physischer und psychischer Abhängigkeit von AAS sind insbesondere nach Absetzen Depressionen, Psychosen und erhöhte Suizidalität beschrieben worden (Pope et al. 2000; Hall et al. 2005). Es ist jedoch unklar, inwieweit psychopathologische Reaktionen Folge des AAS-Abusus oder als zugrundeliegende Störungen mit diesem assoziiert sind.

Nicht zuletzt sind Infektionen und andere Komplikationen durch die Selbstanwendung intramuskulärer Injektionen zu berücksichtigen.

\section{Fazit}

Eine Anwendung von AAS ohne medizinische Indikation ist aus ärztlich-ethischer Sicht strikt abzulehnen. Angesichts der Nebenwirkungen und gravierenden Spätfolgen muß die Verabreichung von AAS zur Leis- 
tungssteigerung an Gesunde berufs- und strafrechtliche Konsequenzen nach sich ziehen. Die frühzeitige Erkennung des AAS-Missbrauchs anhand klinischer Verdachtsmomente sowie die Primärprävention durch intensive Aufklärung insbesondere Jugendlicher sind angesichts der weiten Verbreitung als dringende ärztliche Aufgaben anzusehen.

\section{Literatur}

Bagatell Cl, Bremner WJ (1996) Androgens in men - uses and abuses. N Engl I Med 334, 707-714

Bahrke M, Yesalis CE, Kopstein AN, Stephens JA (2000) Risk factors for anabolic-androgenic steroid use among adolescents. Sports Medicine 29, 1-9

Basaria S (2010) Androgen abuse in athletes: detection and consequences. I Clin Endocrinol Metab 95, 1533-1543

Bhasin S, Storer TW, Berman N, Callegari C, Clevenger B, Phillips I, Bunnel T), Tricker R, Shirazi A, Casaburi R (1996) The effects of supraphysiologic doses of testosterone on muscle size and strength in normal men. $\mathrm{N} \mathrm{Engl} \mathrm{I}$ Med 335, 1-7

Boos C, Wulff P, Kujath P, Bruch H-P (1998) Medikamentenmißbrauch beim Freizeitsportler im Fitneßbereich. Dt Ärztebl 95, A-953-957

Dawson RT (2001) Dugs in sport - the role of the physician. I Endocrinol 170, 55-61

Death AK, McGrath KC, Kazlauskas R, Handelsman DI (2004) Tetrahydrogestrinone is a potent androgen and progestin. I Clin Endocrinol Metab 89, 2498-2500

Duchaine D (1989) Underground steroid handbook II, HLR technical books, Venice, USA

Durant RH, Rickert VI, Ashworth CS, Newman C, Slavens G (1993) Use of multiple drugs among adolescents who use anabolic steroids. N Engl I Med 328, 922-926

Franke WW, Berendonk B (1997) Hormonal doping and androgenization of athletes: a secret program of the German Democratic Republic government. Clinical Chemistry 43, 1262-1279

Geyer H, Parr MK, Mareck U, Reinhart U, Schrader Y, Schänzer W (2004) Analysis of non-hormonal nutritional supplements for anabolic-androgenic steroids - results of an international study. Int / Sports Med 25, 124-129

Hall RC, Hall RC, Chapman MJ (2005) Psychiatric complications of anabolic steroid abuse. Psychosomatics 46, $285-290$

Handelsman D|, Gupta (1997) Prevalence and risk factors for anabolic-androgenic steroid abuse in Australian high school students. Int | Androl 20, 159-164

Karila TA, Karjalainen JE, Mantysaari MJ, Viitasalo MT, Seppala TA (2003) dose-dependant increase in left ventricular mass in power athletes and this effect is potentiated by concomitant use of growth hormone. Int I Sports Med 24, 337-343

Melnik B, Jansen T, Grabbe S (2007) Abuse of anabolic-androgenic steroids and bodybuilding acne: an underestimated health problem. J Dtsch Dermatol Ges 5, 110-117

Mottram DR, George Al (2000) Anabolic steroids. Baillieres Best Pract Res Clin Endocrinol Metab 14, 55-69

Parkinson AB, Evans NA (2006) Anabolic androgenic steroids: a survey of 500 users. Med Sci Sports Exerc 38, $644-651$

Petersson A, Bengtsson I, Voltaire-Carlsson A, Thiblin I (2010) Substance abusers' motives for using anabolic androgenic steroids. Drug Alcohol Depend 111, 170-172

Pope HG, Phillips KA, Olivardia R (2000) The Adonis Complex: the secret crisis of male body obsession. Free Press, New York

Schänzer W (2004) Abuse of androgens and detection of illegal use. In: Nieschlag E, Behre HM (eds.) Testosterone - action, deficiency, substitution, 3rd ed. Cambridge University Press, Cambridge, S. 715-735

Sjöqvist F, Garle M, Rane A (2008) Use of doping agents, particularly anabolic steroids, in sports and society. Lancet 371, 1872-1882

Striegel H, Simon P, Frisch S, Roecker K, Dietz K, Dickhuth HH, Ulrich R (2006) Anabolic ergogenic substance users in fitness-sports: A distinct group supported by the health care system. Drug Alcohol Depend 81, 11-19

Verroken M (2000) Drug use and abuse in sport. Baillieres Best Pract Res Clin Endocrinol Metab 14, 1-23

Wade N (1972) Anabolic steroids: doctors denounce them but athletes aren't listening. Science 176, 1399-1403 